\title{
Ecological Models Explaining the Success of Distinctive Sperm and Eggs (Oogamy)
}

\author{
DAVID B. DusEnBery* ${ }^{*}$ \\ $\dagger$ School of Biology, Georgia Institute of Technology, 310 Ferst Drive, Box 0230, \\ Atlanta, GA 30332-0230, U.S.A.
}

(Received on 13 December 2001, Accepted in revised form on 3 June 2002)

\begin{abstract}
Several lineages have independently evolved from isogamy (all sexes producing similar gametes) through anisogamy (dissimilar gametes) to the familiar male (producing sperm) and female (producing eggs) condition of most large, multicellular organisms (oogamy). A variety of hypotheses explaining the selective mechanisms causing such evolution and the success of these lineages have been proposed, but little evidence and some confusion persists. Here, a few simplifying assumptions are used to extract and compare the essential features of the various ecological hypotheses. The comparisons reveal that the critical need is to identify a selective advantage of large, immobile gametes (eggs). Assumptions about the effect of sperm size on swimming speed are not important. The classic assumption of increasing zygote success with large size requires a relationship even stronger than survival proportional to volume, which seems unlikely and lacks empirical support. An assumption that eggs produce a pheromone sperm attractant leads, by established physical principles, to a more than sufficient advantage of large egg size. Without pheromones, combinations of increased target size and weaker increased zygote fitness or increased gamete longevity also provide sufficient selection.
\end{abstract}

(C) 2002 Elsevier Science Ltd. All rights reserved.

\section{Introduction}

One of the most interesting questions in evolution is what adaptive pressures led to the widespread success of sexual reproduction. The answer is still not clear (Agrawal, 2001; Barton \& Charlesworth, 1998; Crow, 1994; Halvorson \& Monroy, 1985; Hastings, 1999; Roughgarden, 1991; Siller, 2001). The observation that many species of algae and fungi carry out sexual reproduction by fusing gametes of the same size (isogamy) raises the related question of why

\footnotetext{
*Corresponding author. Tel.: + 1404894 8426; fax: +14048940519.

E-mail address: david.dusenbery@biology.gatech.edu (D.B. Dusenbery).
}

male-female mating systems are so successful that almost all large, multicellular organisms have males and females (defined by the production of numerous, small, dispersing gametes and few, large, stationary gametes).

It appears that several lineages have independently progressed from isogamy to anisogamy (fertilization by fusing gametes of different size) to oogamy (fertilization by fusing large, immobile eggs and small, mobile sperm) to internal fertilization, suggesting that there are general selective pressures at work (Baccetti, 1985; Margulis \& Sagan, 1986). The last step to internal fertilization can be understood as contributing to independence from liquid water among terrestrial organisms and providing a 
variety of other benefits for large, mobile animals. But the earlier steps are more difficult to explain.

One type of explanation that has been explored is based on conflict between cytoplasmic elements of the two gametes. However, in a recent review, Randerson \& Hurst (2001) conclude that this explanation is not satisfactory.

A variety of other explanations have been proposed over several decades (Charlesworth, 1978; Cox \& Sethian, 1985; Dusenbery, 2000; Hoekstra, 1984; Hoekstra et al., 1984; Parker et al., 1972; Schuster \& Sigmund, 1982; Scudo, 1967), but disagreement about their plausibility and misunderstanding of their underlying assumptions continues. Most alternative explanations are ecological in the sense that they focus on allocation of resources, selection on size, and/or behavioral interactions between gametes. Here, I develop an approach to analyse and compare these models by extracting their essential size-dependent components of selection in a common form. This allows clear comparisons of their fundamental features and suggests several new possible explanations.

\section{Measure of Fitness}

In quantitative models, most authors assume that adults of both sexes have equal total volumes $\left(V_{T}\right)$ of cytoplasm available to form gametes. The evolutionary choice is how many $\left(n_{G}\right)$ equal gametes should this volume be divided among. The volume of each gamete $\left(V_{G}\right)$ is inversely related to the number of gametes: $V_{G}=V_{T} / n_{G}$. Beyond this assumption, authors diverge in their assumptions as to which factors are influenced by gamete size and how the factor depends on size. By making a few assumptions, the several hypotheses can be put in a common framework that facilitates comparison.

Assume that evolutionary success depended on survival of crises when population densities were low. At these times, populations faced sperm limitation, and survival depended on the effectiveness with which appropriate gametes came together.
Furthermore, assume (as have others) that the important features can be captured by making the simplifying assumptions that gametes can be approximated as spheres and have a uniform probability of occurrence in three-dimensional space. Such a random distribution is most likely to occur as a result of turbulent currents. Although such distributions are unlikely to occur over wide areas, they are likely at the small spatial scales were gametes encounter each other. Consequently, we will consider population densities, and $V_{T}$ is interpreted as the total volume of each type of gamete per unit volume of the environment.

With these assumptions, the following quantitative description can be developed. For simplicity of presentation, consider a population spawning synchronously once per generation. (A continuously reproducing model could have very similar mathematical structure and conclusions.) Reproductive rate is broken down into the product of three components: the rate at which encounter is made between gametes of opposite mating type ( $R$, in number per second per unit volume of the environment); the time span over which gametes are effective ( $t$, in seconds); and the fraction of encounters that result in a zygote that survives and develops into a reproductive adult $(f)$.

Assuming also that oogamy is already established, the situation is further simplified. For immobile eggs $(E)$ and motile sperm $(S), R$ depends on the effective target size of the egg ( $\sigma_{E}=$ cross-sectional area) and swimming velocity of the sperm $\left(v_{S}\right)$, as well as the number density of the two kinds of gametes $\left(n_{E}, n_{S}\right)$. For a spherical egg with radius $r_{E}, \sigma_{E}=\pi r_{E}^{2}$. For a gamete of radius $r_{G}, V_{G}=(4 \pi / 3) r_{G}^{3}$ and the density is $n_{G}=V_{T} / V_{G}=(3 / 4 \pi) V_{T} r_{G}^{-3}$. Assuming that the swimming path is straight over distances greater than the target size, many analyses (Cox \& Sethian, 1985; Dusenbery, 1992, pp. 386-390; Gerritsen \& Strickler, 1977; Kauzmann, 1966, eqn 5-15) demonstrate that $R=n_{E} n_{S} \sigma_{E} v_{S}=\left(V_{T} / V_{E}\right)\left(V_{T} / V_{\mathrm{S}}\right) \sigma_{E} v_{S}$.

Growth of a population requires that the product Rtf exceed the density of reproductive adults. Thus, fitness could be taken as the product of six parameters: $n_{S} n_{E} \sigma_{E} v_{S} t f$. However, it is more specific to eliminate $V_{T}$, which 
is assumed maximized in all cases of a given population density, and we get the fitness measure

$$
w=\frac{R t f}{V_{T}^{2}}=\frac{\sigma_{E}}{V_{E}} \frac{v_{S}}{V_{S}} t f .
$$

We now see that fitness is maximized by increasing the ratio of target cross section to volume of the egg, the ratio of speed of swimming to volume of the sperm, the active time period of both gametes, and the fraction of encounters that give rise to reproducing adults.

To see how this fitness relates to the minimum population size, define $p$ as the productivity of adults in producing gamete volume, $V_{T}=p n_{A}$. Then, in generation $\tau+1, n_{A}(\tau+1)=R t f=$ $w V_{T}^{2}=w p^{2} n_{A}(\tau)^{2}$. Population stability requires $n_{A}(\tau+1) \geqslant n_{A}(\tau)$ and the minimum population limit is $n_{A}(\tau) \geqslant 1 / w p^{2}$. Increasing gamete productivity has a large effect. But, for any given productivity, it is also beneficial to increase $w$, the measure of fitness used here. The main goal of the present investigation is to identify plausible hypotheses for why $w$ is higher for populations in which the two kinds of gametes are very different in size.

\section{Approximations of Models}

Equation (1) provides a foundation for comparing all the relevant models that have been proposed. Although the models are diverse and often involve complicated mathematical expressions, each of these parameters is proportional to some power of the radius of sperm or egg, at least in the limits of extreme dimorphism $\left(r_{E} \gg r_{S}\right)$ and oogamy $\left(v_{E}=0\right)$. Since the fitness defined by eqn (1) is simply the product of six parameters or their reciprocals, the exponents from all the parameters can be combined and provide a concise measure of the strength of the influence of egg and sperm size on fitness. It is this strength that is critical.

I will use " $\propto$ " to symbolize proportionality.

Scudo (1967) developed a basic mathematical model of gamete encounter probabilities, with an emphasis on the time period for which the gametes are functional. However, for fitness he focused on the efficiency of gamete use rather than the probability of fertilization, which is the focus here and in most other works. Also, we focus on what happens at the limits of low density and can discard the complications he used to deal with multiple encounters.

The first model to receive much attention was proposed in Parker et al., 1972. The authors assumed that zygote success was proportional to zygote volume (equal to the sum of gamete volumes) raised to some power, $x$, which is a constant determined by the environment. In the symbols used here,

$$
\begin{aligned}
f & \propto\left(V_{E}+V_{S}\right)^{x}=\left[(4 \pi / 3) r_{E}^{3}+(4 \pi / 3) r_{S}^{3}\right]^{x} \\
& =(4 \pi / 3)^{x}\left(r_{E}^{3}+r_{S}^{3}\right)^{x} \\
& \propto\left(r_{E}^{3}+r_{S}^{3}\right)^{x} .
\end{aligned}
$$

Since $r_{E} \gg r_{S}, r_{E}^{3}+r_{S}^{3}$ can be approximated by $r_{E}^{3}$, and the assumption reduces to $f \propto r_{E}^{3 x}$. Their analysis indicated that if $x$ was in the approximate range $1.8<x<2.5$ anisogamy was selected (their Figs 5-7). I used $x=2\left(f \propto r_{E}^{6}\right)$ for the example in the column labeled "Zygote*", in Table 1.

Charlesworth (1978) analysed more rigorously a similar model, concluding that $x>1$ was sufficient (his equation 7a). So $f \propto r_{E}^{>}$is used in Table 1-Zygote†.

Schuster \& Sigmund (1982) added to the modern literature the idea that gamete encounter rates are important and provided calculations assuming that non-mobile gametes encountered each other as the result of Brownian motion. Their equation 1 is equivalent to assuming that the product $\sigma_{E} v_{S}=\left(r_{E}+r_{S}\right)^{2} r_{E}^{-1} r_{S}^{-1}$. In the limit, $r_{E} \gg r_{S}$, this reduces to the approximation $\sigma_{E} v_{S} \approx r_{E} r_{S}^{-1}$, which is used in Table 1Diffusion.

Hoekstra et al. (1984) analysed a model without any dependence of zygote fitness on size. Instead they assumed that fitness was dominated by encounter frequency, which was aided by pheromone attractants acting between all gametes. The primary effect they relied on was a reduction of the pheromone active space with increasing speed of swimming. This effect is 
TABLE 1

Comparison of anisogamyloogamy models

\begin{tabular}{|c|c|c|c|c|c|c|c|c|}
\hline Parameter & Zygote* & Zygote $\dagger$ & Diffusion & Hoekstra§ & Search $\|$ & Pheromone & Target ${ }^{* * *}$ & Longevity ${ }^{* *}$ \\
\hline$n_{E}$ or $V_{E}^{-1}$ & $r_{E}^{-3}$ & $r_{E}^{-3}$ & $r_{E}^{-3}$ & $r_{E}^{-3}$ & $r_{E}^{-3}$ & $r_{E}^{-3}$ & $r_{E}^{-3}$ & $r_{E}^{-3}$ \\
\hline$n_{S}$ or $V_{S}^{-1}$ & $r_{S}^{-3}$ & $r_{S}^{-3}$ & $r_{S}^{-3}$ & $r_{S}^{-3}$ & $r_{S}^{-3}$ & $r_{S}^{-3}$ & $r_{S}^{-3}$ & $r_{S}^{-3}$ \\
\hline $\begin{array}{l}\sigma_{E} \\
v_{S}\end{array}$ & - & - & \}$r_{E} r_{S}^{-1}$ & $\begin{array}{l}r_{E}^{0} \\
r_{S}^{-2}\end{array}$ & $\begin{array}{l}r_{E}^{2} \\
r_{S}^{-1}\end{array}$ & $\begin{array}{l}r_{E}^{6} \\
r_{S}\end{array}$ & $\underline{r_{E}^{2}}$ & $r_{E}^{2}$ \\
\hline$t$ & $\overline{x^{6}}$ & $\bar{r}>3$ & $\bar{r}>2$ & & & - & - & $r_{E}^{2} r_{S}^{2}$ \\
\hline$f$ & $r_{E}^{\sigma}$ & $r_{E}^{-}$ & $r_{E}^{2}$ & $r_{E}^{3}$ & $r_{E}^{3}$ & - & $r_{E}^{>1}$ & \\
\hline Product & $r_{E}^{3} r_{S}^{-3}$ & $r_{E}^{>0} r_{S}^{-3}$ & $r_{E}^{>0} r_{S}^{-4}$ & $r_{E}^{>0} r_{S}^{-5}$ & $r_{E}^{2} r_{S}^{-4}$ & $r_{E}^{3} r_{S}^{-2}$ & $r_{E}^{>0} r_{S}^{-3}$ & $r_{E} r_{S}^{-1}$ \\
\hline
\end{tabular}

Note. Subscript $E$ refers to eggs, $S$ to sperm. $r^{>x}$ is gamete radius to any exponent greater than $x$. The product of the six parameters is the measure of fitness, in this situation. The relations are taken from the models in the limits $r_{E} \gg r_{S}, v_{E}=0$.

* Parker et al. (1972).

$\dagger$ Charlesworth (1978).

\$ Schuster \& Sigmund (1982) did not work out how much smaller the zygote exponent could be, but this analysis indicates that it can get down close to 2 .

$\S$ Hoekstra (1984).

|Cox \& Sethian (1985).

T Dusenbery (2000).

*** Proposed here.

well documented (Dusenbery, 1992, pp. 71-74), but the resulting mathematics are too complicated to solve analytically. In addition, they ignore the serious difficulties of simultaneously releasing a pheromone yourself and detecting that the same pheromone has been released by another source. Given these problems, I neglect this model.

Hoekstra (1984) analysed similar models. In this case, he assumed that fitness was proportional to swimming speed, which was in turn inversely proportional to the cross-sectional area $\left(v_{S} \propto r_{S}^{-2}\right)$. One of his models (his Section 5) made the zygote selection on size assumption of Parker et al. (1972), $f \propto V_{Z}^{x} \propto r_{Z}^{3 x}$, and his Fig. 2 indicates $x$ must exceed 1 , as Charlesworth found. This model is summarized in Table 1Hoekstra.

Cox \& Sethian (1985) also assumed that encounter frequency was critical. Deriving the relation for encounter rate among spheres moving in straight lines, they obtained their equation 14 , which can be simplified to the more common form for the encounter rate (e.g. Kauzmann, 1966, eqn 5-15; Gerritsen \& Strickler, 1977), assuming $v_{\mathrm{S}} \geqslant v_{E}$ :

$$
R=\pi n_{E} n_{S}\left(r_{E}+r_{S}\right)^{2}\left(3 v_{S}^{2}+v_{E}^{2}\right) / 3 v_{S}
$$

For oogamy, $v_{E}=0$ and $r_{E} \gg r_{S}$, these assumptions give us the approximation

$$
R \approx \pi n_{E} n_{S} r_{E}^{2} v_{S}
$$

They further assumed that gametes are propelled at constant thrust, independent of size. Under the appropriate conditions (low Reynolds number) it is well established (Stokes' law) that, at constant thrust, speed is inversely proportional to size $\left(v_{S} \propto r_{S}^{-1}\right)$. Thus, their model includes the fundamental relation

$$
R \propto n_{E} n_{S} r_{E}^{2} r_{S}^{-1} .
$$

With their assumption that $f \propto r_{E}^{3}$, their model has fitness

$$
w=R t f \propto R f \propto r_{E}^{-3} r_{S}^{-3} r_{E}^{2} r_{S}^{-1} r_{E}^{3} \propto r_{E}^{2} r_{S}^{-4}
$$

This relation is included in Table $1-$ Search.

Dusenbery (2000) adopted a similar model but discarded the assumption that zygote size contributed to fitness and added the assumption that eggs produce a pheromone sperm attractant. This assumption dramatically increased the target size of the egg. Assuming that the resources available for pheromone production or propulsion are proportional to gamete volume and that diffusion is the only mechanism 
of pheromone transport, a detailed model, including recognition of the volume of cargo (chromosomes) that a gamete must carry, was developed. For oogamy, his equation 3 is equivalent to

$$
R \propto r_{S}^{-3} r_{E}^{-3}\left(r_{S}+r_{P}\right)^{2}\left(r_{S}^{2}-r_{S}^{-1}\right)^{1 / 2},
$$

where $r_{P}$ is the radius of the active space of the pheromone. With the assumption of diffusion, the radius out to the detection threshold concentration is proportional to the rate of pheromone production, which is proportional to the volume of the egg minus the volume occupied by the genetic cargo, giving $r_{P}=r_{E}^{3}-1$. The size scale here is the minimum radius necessary to enclose the chromosome volume, and $r_{P} \geqslant r_{E} \gg r_{S}>1$. The approximations give $r_{S}+r_{P} \approx r_{P} \propto r_{E}^{3}$ and $r_{S}^{2}-r_{S}^{-1} \approx r_{S}^{2}$, which lead to

$$
w=R t f \propto R \propto r_{S}^{-3} r_{E}^{-3} r_{E}^{6} r_{S}=r_{E}^{3} r_{S}^{-2},
$$

which is included in Table 1 -Pheromone. In this model, pheromone production and power applied to propulsion are both proportional to volume. At low Reynolds number, the latter assumption results in speed directly proportional to linear size, $v_{S} \propto r_{S}$ (Dusenbery \& Snell, 1995).

\section{Results}

The fundamental relations for fitness dependence on egg and sperm size have been extracted from each of the published models and presented in Table 1.

For the disruptive selection on gamete size necessary to favor oogamy over isogamy, the fitness must have a positive exponent on egg size and a negative exponent on sperm size, so that fitness increases with larger eggs and smaller sperm (over at least some size range). Since the number of gametes $\left(n_{G}\right)$ provides all models with a negative three exponent for both gametes, it alone provides strong selective pressure for small sperm size, and assumptions about sperm swimming speed are not critical. The challenge is to find a selective pressure that favors large egg size sufficient to overcome the loss of numbers with large size. This notion is consistent with the view that the isogamy-to-oogamy transition involved the growth of egg size more than the shrinkage of sperm (Bell, 1997).

The classical solution is to assume that zygote fitness $(f)$ increases strongly with zygote volume $\left(V_{Z}\right)$. However, it is easy to see from the analysis presented here that $f$ must be more than proportional to volume $\left(r_{E}^{>3}\right)$, since gamete number declines in proportion to volume. The first analysis (Parker et al., 1972) suggested $f \propto r_{E}^{6}$ was sufficient (Table 1-Zygote*), while a subsequent (more rigorous) analysis (Charlesworth, 1978) indicated $f \propto r_{E}^{>3}$ is sufficient (Table 1-Zygote $\dagger$ ). The latter constraint corresponds with the proposed requirement of a positive exponent on egg size for the overall fitness.

Schuster and Sigmund (1982) added a gameteencounter mechanism based on Brownian motion, without any motile gametes. From Table 1 -Diffusion, it is seen that this reduces the required size dependence of zygote success from greater than proportional to volume $\left(r_{E}^{>3}\right)$ to greater than proportional to area $\left(r_{E}^{>2}\right)$, which is a more plausible possibility.

Hoekstra (1984) adds the assumption that $v_{S} \propto r_{S}^{-2}$ to the gamete number assumptions. Table 1 -Hoekstra, reveals that this assumption accomplishes little, as it does not contribute to selection for large eggs. And it is still necessary to assume that zygote success is more than proportional to volume.

Cox \& Sethian (1985) emphasize the advantage of increased target size with larger eggs. When this is combined with the assumption that zygote success is proportional to volume, the required disruptive selection occurs (Table $1-$ Search).

More recently, Dusenbery (2000) proposed a detailed physical model based on the assumption that eggs produce a pheromone sperm attractant. This provides a mechanism to dramatically increase their target size. Assuming diffusion of pheromone, the radius of the pheromone active space is proportional to the rate of pheromone production, and this radius is squared to get the target size. Assuming the resources available to produce pheromone are proportional to egg volume $\left(r_{E}^{3}\right)$, target area varies as the sixth power of egg radius (square of volume), and this provides a powerful selective force for large 
eggs. The results in Table $1-$ Pheromone, also indicate that the required selection would still occur if the assumption were relaxed to pheromone production proportional to egg surface area $\left(r_{E}^{2}\right)$. This model provides the required selective force without any assumption that zygote success depends on size.

\section{Discussion}

These simplifying assumptions allow essential features of the diverse models to be extracted and compared, permitting identification of the critical differences between existing models and identification of other possible hypotheses.

Although it is plausible that zygote fitness would increase with size, there is little data supporting this assumption (Madsen \& Waller, 1983; Randerson \& Hurst, 2001), and it is doubtful that the relation is stronger than proportional to volume $\left(w \propto r_{Z}^{>}\right)$as required if this is the only selective force at work. Proportional to area $\left(w \propto r_{Z}^{2}\right)$ or linear size $\left(w \propto r_{Z}\right)$ would be increasingly more plausible. The required relation is reduced in models also assuming that larger eggs represent a larger target for sperm (Table 1-Diffusion, - Search, and - Target models). This makes the models more plausible but still retains the ad hoc assumption that larger zygotes are more fit.

Assumptions about sperm swimming speed have received more attention than deserved. From the analysis presented here, it is clear that no assumption about sperm swimming speed can provide selection for large eggs. Contrary claims (Hoekstra, 1984, 1987) are based on unreasonably throwing out the volume/number trade-off for gametes. It has been argued (Dusenbery, 2000; Dusenbery \& Snell, 1995) that the best assumption for the relation between swimming speed and sperm size is direct proportionality, but this assumption has been criticized (Randerson \& Hurst, 2001). In any case, it is clear that this assumption is not necessary in the pheromone model (Table 1-Pheromone; Dusenbery, 2000). In fact, Dusenbery's assumption makes selection for dimorphism more difficult. Any of the other assumptions used for $v_{S}$ in any of these models increases the strength of selection for dimorphism.
The analysis presented here makes it easy to see alternative models that have not previously been proposed. A straightforward prediction of search theory leads to the expectation that target size is proportional to egg cross-sectional area $\left(\sigma_{E} \propto r_{E}^{2}\right)$ (Cox \& Sethian, 1985; Dusenbery, 1992, pp. 386-390). This assumption provides the necessary selection, if it is combined with an assumption that zygote success is more than proportional to linear size $\left(f \propto r_{E}^{>1}\right)$ (Table $1-$ Target), which is much more plausible than proportional to volume $\left(f \propto r_{E}^{>}\right)$.

Alternatively, consider a population spawning continuously, producing $C$ volumes of gametes per second. If gametes of mating type $G$ are active for $t_{G}$ seconds, then we have $n_{G}=C t_{G} / V_{G}$ in comparison to the previous relation $n_{G}=$ $V_{T} / V_{G}$ for synchronous populations. If we now assume that the active time period of a gamete is proportional to its surface area $\left(t_{G} \propto r_{G}^{2}\right)$ then $n_{G} \propto r_{G}^{-1}$. As shown in Table $1-$ Longevity, when combined with the standard target-size assumption, this assumption of gamete longevity is sufficient to provide the required disruptive selection, and longevity proportional to volume would work even better.

At present, the most plausible selective force seems to be provided by the pheromone model (with any of several possible speed assumptions), since the eggs of many species are known to release sperm attractants (Hoekstra, 1987; Hoekstra et al., 1984). Also it provides the strongest selection for larger eggs, and the excess selective pressure $\left(r_{E}^{3}\right.$ where $r_{E}^{>0}$ is needed) can accommodate weaker relationships than assumed in the calculation. For zygote size selection to be as strong, fitness must be proportional to the square of zygote volume (sixth power of radius), which seems extremely unlikely. Most of these mechanisms are not mutually exclusive, and size benefits of gamete longevity, and zygote success could contribute to selection, although unlikely to be sufficient on their own.

It should be pointed out that these models are not all at the same level of abstraction. The assumption of selection on zygote size has no mechanistic theory identifying a selective agent or empirical support; the emphasis of the authors was on population genetics. The proposed selection on gamete longevity has similar 
status. In contrast, most of the other models provide mechanistic theories with specified selective agents; the emphasis is on identifying the selective forces at work - often well-established constraints of physics. Future work should aim to combine the two approaches to provide a mechanistically plausible selective force with population genetics models.

It is useful to note that there are two distinct questions regarding the evolution of oogamy. One is: How did oogamy evolve in the first place? This question is addressed more by population genetics approaches, but the present analysis should help identify selective forces. Whatever the mechanism, the evidence indicates that oogamy evolved independently in several lineages (Baccetti, 1985; Margulis \& Sagan, 1986). The second question is: Why have lineages with oogamy been so successful in producing large organisms? The analysis presented here bares more clearly on this question, especially if it is assumed that longevity of the lineage (ability to survive population crashes) was a critical factor necessary to provide the time to evolve multicellularity and the physiological systems (circulation, gas exchange, etc.) necessary for large body size.

I thank an anonymous reviewer for thoughtful comments and suggestions for improving the manuscript.

\section{REFERENCES}

Agrawal, A. F. (2001). Sexual selection and the maintenance of sexual reproduction. Nature 411, 692-695.

BACCETTI, B. (1985). Evolution of the sperm cell. In: Biology of Fertilization (Metz, C. B. \& Monroy, A., eds), Vol. 2, pp. 3-58. Orlando: Academic Press.

Barton, N. H. \& Charlesworth, B. (1998). Why sex and recombination. Science 281, 1986-1990.

Bell, G. (1997). The evolution of the life cycle of brown seaweeds. Biol. J. Linnean Soc. 60, 21-38.
Charlesworth, B. (1978). The population genetics of anisogamy. J. theor. Biol. 73, 347-357.

Cox, P. A. \& Sethian, J. A. (1985). Gamete motion, search, and the evolution of anisogamy, oogamy, and chemotaxis. Am. Nat. 125, 74-101.

Crow, J. F. (1994). Advantages of sexual reproduction. Dev. Genet. 15, 205-213.

Dusenbery, D. B. (1992). Sensory Ecology. New York: W. H. Freeman and Company.

Dusenbery, D. B. (2000). Selection for high gamete encounter rates explains the success of male and female mating types. J. theor. Biol. 202, 1-10.

Dusenbery, D. B. \& Snell, T. W. (1995). A critical body size for use of pheromones in mate location. J. Chem. Ecol. 21, 427-438.

Gerritsen, J. \& Strickler, J. R. (1977). Encounter probabilities and community structure in zooplankton: a mathematical model. J. Fish. Res. Board Can. 34, 73-82.

Halvorson, H. O. \& Monroy, A. (1985). The Origin and Evolution of Sex. New York: Alan R. Liss.

Hastings, I. M. (1999). The costs of sex due to deleterious intracellular parasites. J. Evol. Biol. 12, 177-183.

Hoekstra, R. F. (1984). Evolution of gamete motility differences II. Interaction with the evolution of anisogamy. J. theor. Biol. 107, 71-83.

Hoekstra, R. F. (1987). The evolution of sexes. In: The Evolution of Sex and its Consequences (Stearns, S. C., ed.), pp. 59-91. Basel: Birkhäuser Verlag.

Hoekstra, R. F., Janz, R. F. \& Schilstra, A. J. (1984). Evolution of gamete motility differences I. Relation between swimming speed and pheromonal attraction. J. theor. Biol. 107, 57-70.

Kauzmann, W. (1966). Kinetic Theory of Gases. New York: W. A. Benjamin, Inc.

Madsen, J. D. \& Waller, D. M. (1983). A note on the evolution of gamete dimorphism in algae. Am. Nat. 121, 443-447.

Margulis, L. \& Sagan, D. (1986). Origins of Sex. New Haven, CT: Yale University Press.

Parker, G. A., Baker, R. R. \& Smith, V. G. F. (1972). The origin and evolution of gamete dimorphism and the male-female phenomenon. J. theor. Biol. 36, 529-553.

Randerson, J. P. \& Hurst, L. D. (2001). The uncertain evolution of the sexes. Trends Ecol. Evol. 16, 571-579.

Roughgarden, J. (1991). The evolution of sex. Am. Nat. 138, 934-935.

Schuster, P. \& Sigmund, K. (1982). A note on the evolution of sexual dimorphism. J. theor. Biol. 94, 107-110.

Sudo, F. M. (1967). The adaptive value of sexual dimorphism: I, anisogamy. Evolution 21, 285-291.

Siller, S. (2001). Sexual selection and the maintenance of sex. Nature 411, 689-692. 